\title{
On inequalities associated with the Jordan-von Neumann functional equation
}

JÜRG RÄTZ

Summary. For a group $(G, \cdot)$ and a real or complex inner product space $(E,\langle\cdot, \cdot\rangle)$ with norm 『.』 we consider the functional inequality

$$
f: G \longrightarrow E, \llbracket 2 f(x)+2 f(y)-f\left(x y^{-1}\right) \rrbracket \leq \rrbracket f(x y) \rrbracket \quad(\forall x, y \in G)
$$

and describe situations in which (I) implies the Jordan-von Neumann parallelogram equation

$$
f: G \longrightarrow E, 2 f(x)+2 f(y)=f(x y)+f\left(x y^{-1}\right) \quad(\forall x, y \in G) .
$$

Mathematics Subject Classification (2000). 39B62.

Keywords. Functional inequalities, functional equations, quadratic functions, inner product spaces.

\section{Introduction}

Throughout the paper, $G$ or $(G, \cdot)$ or $(G, \cdot, e)$ will denote a (not necessarily abelian) group with group operation - and identity element $e$ and $E$ or $(E,\langle\cdot, \cdot\rangle)$ an inner product space over $\mathbb{K}(=\mathbb{R}$ or $\mathbb{C})$ with inner product $\langle\cdot, \cdot\rangle$ and associated norm $\rrbracket \cdot \rrbracket$.

Starting from the Jordan-von Neumann functional equation $(\mathrm{JvN})$, fourteen inequalities may be obtained by first transferring at most one summand of either side of $(\mathrm{JvN})$ to the other, then taking norms, and finally replacing $=$ by $\leq$ or $\geq$. Of course, each of these inequalities is a weakening of $(\mathrm{JvN})$. The question is whether this weakening is always strict. In [4] (p. 304, Satz 1; p. 307, Bemerkungen 1 und 2), A. Gilányi comes to the quite surprising conclusion that exactly one of these inequalities (namely (I) above) is equivalent to $(\mathrm{JvN})$. The main result of [4] reads as follows:

Theorem 1.1. (A. Gilányi).

a) If $(G, \cdot, e)$ is a 2 -divisible abelian group and $(E,\langle\cdot, \cdot\rangle)$ an inner product space over $\mathbb{K}$, then (I) implies $(\mathrm{JvN})$, i.e., $f$ is quadratic.

b) In part a), the commutativity of $G$ may be replaced by the condition

$$
f(x y z)=f(x z y) \quad(x, y, z \in G) .
$$


It is the purpose of this paper to show that 2-divisibility of $G$ can be deleted and that $(\mathrm{C})$ can be essentially weakened and to exhibit classes of examples of groups in which we do not have to care about any commutation condition. The results were announced in [5] and [12], and variants of Theorem 1.1 were discussed on the 39th International Symposium on Functional Equations (cf. [6], [12]). The question as to whether in the general case, some substitute for commutativity is needed for $(\mathrm{I}) \Longrightarrow(\mathrm{JvN})$ remains open.

Further notations. The symbol $:=$ means that the right hand side defines the left hand side. The symbols $\mathbb{N}, \mathbb{N}^{0}, \mathbb{Z}, \mathbb{Q}, \mathbb{R}, \mathbb{C}$ denote the sets of positive integers, nonnegative integers, integers, rational, real, and complex numbers, respectively; $\mathbb{R}_{+}:=\{\alpha \in \mathbb{R} ; \alpha \geq 0\}, \mathbb{R}_{+}^{*}:=\{\alpha \in \mathbb{R} ; \alpha>0\}$. $\mathbb{K}$ stands for $\mathbb{R}$ or $\mathbb{C}$, and $\mathbb{K}^{*}:=\{\alpha \in \mathbb{K} ; \alpha \neq 0\}$. We denote by $\underline{c}$ the constant mapping with value $c$, and by $i_{B}$ the identity mapping of the set $B$. For an easy unified treatment of the cases $\mathbb{K}=\mathbb{R}$ and $\mathbb{K}=\mathbb{C}$, we put for $\mathbb{K}=\mathbb{R}: \operatorname{Re}:=i_{\mathbb{R}}, \operatorname{Im}:=\underline{0}$. We use 0 for the zero vector of $E$ as well as for the number zero and for the identity element of an additively written abelian group; it will always be clear from the context what is meant.

\section{Substitutes for commutativity of $G$}

Remark 2.1. To the author's knowledge, the first occurrence of condition (C) (cf. Theorem 1.1 b) above) is in PL. Kannappan's Ph.D. Thesis work where it plays a fundamental role in the theory of d'Alembert's functional equation (cum grano salis also called the "cosine equation", [9], p. 72, Theorem 2).

Remark 2.2. Let for the moment $M$ be a nonempty set and $f: G \longrightarrow M$.

a) (C) turns out to be equivalent to the invariance of $f\left(z_{1} \cdot \ldots \cdot z_{n}\right)(n \in \mathbb{N}$, $\left.n \geq 2 ; z_{1}, \ldots, z_{n} \in G\right)$ under all permutations of the factors $z_{1}, \ldots, z_{n}$. Therefore (C) implies each of the three conditions

$$
\begin{array}{rlrl}
f(x y) & =f(y x) & & (\forall x, y \in G), \\
f\left(x y x y^{-1}\right) & =f\left(x^{2}\right) & & (\forall x, y \in G), \\
f\left(y^{-1} x^{-1} y x^{-1}\right) & =f\left(x^{-2}\right) & (\forall x, y \in G) .
\end{array}
$$

By the way, $\left(\mathrm{C}^{\prime}\right)$ is equivalent to the invariance of $f\left(z_{1} \cdot \ldots \cdot z_{n}\right)(n \in \mathbb{N}$, $\left.n \geq 2 ; z_{1}, \ldots, z_{n} \in G\right)$ under all circular rearrangements (i.e., under all powers of the index permutation $(12 \ldots n))$ of the factors $z_{1}, \ldots, z_{n}$.

b) It is easily seen that in case of an even mapping $f: G \longrightarrow M$ (i.e., $f\left(x^{-1}\right)=$ $f(x)(\forall x \in G)),\left(\mathrm{C}^{\prime \prime}\right)$ and $\left(\mathrm{C}^{\prime \prime \prime}\right)$ are equivalent. 
Vol. 66 (2003) Inequalities associated with the Jordan-von Neumann functional equation 193

\section{Variants of Theorem 1.1}

A useful tool for later purposes will be

Lemma 3.1. For elements $a, b$ of the inner product space $E$

$$
\llbracket a \rrbracket^{2} \leq \operatorname{Re}\langle a, b\rangle
$$

and

$$
\llbracket a \rrbracket=\rrbracket b \rrbracket
$$

imply $a=b$.

Proof. We have $\llbracket a-b \rrbracket^{2}=\llbracket a \rrbracket^{2}-2 \operatorname{Re}\langle a, b\rangle+\llbracket b \rrbracket^{2}={ }_{(2)}=2 \rrbracket a \rrbracket^{2}-2 \operatorname{Re}\langle a, b\rangle \leq{ }_{(1)} \leq 0$, so $a=b$.

The next theorem strengthens Theorem 1.1 by deleting the divisibility assumption and by weakening the commutativity requirement for $G$.

Theorem 3.2. Let $(G, \cdot, e)$ be an arbitrary group, $(E,\langle\cdot, \cdot\rangle)$ an inner product space over $\mathbb{K}$, and $f: G \longrightarrow E$ a solution of $(\mathrm{I})$. Then we have

a) $f(e)=0, f\left(x^{-1}\right)=f(x), f\left(x^{2}\right)=4 f(x)(\forall x \in G)$.

b) If $f$ satisfies $\left(\mathrm{C}^{\prime \prime}\right)$ (or, equivalently, $\left(\mathrm{C}^{\prime \prime \prime}\right)$ ), then $f$ is quadratic.

Proof. a) We first follow the proof of ([4], Satz 1) to obtain

$$
f(e)=0
$$

and

$$
2 f(x)+2 f\left(x^{-1}\right)=f\left(x^{2}\right) \quad(\forall x \in G)
$$

and now deviate from it. Taking $x \in G$ arbitrarily, putting $y:=x$ in (I) and using (3) we get

$$
\llbracket 4 f(x) \rrbracket \leq \llbracket f\left(x^{2}\right) \rrbracket \quad(\forall x \in G) .
$$

Again let $x \in G$ be arbitrary. Then $\llbracket 4 f(x) \rrbracket \leq{ }_{(5)} \leq \llbracket f\left(x^{2}\right) \rrbracket=(4)=\llbracket 2 f(x)+$ $2 f\left(x^{-1}\right) \rrbracket \leq \llbracket 2 f(x) \rrbracket+\llbracket 2 f\left(x^{-1}\right) \rrbracket$, so $\llbracket 2 f(x) \rrbracket \leq \llbracket 2 f\left(x^{-1}\right) \rrbracket$, i.e. $\llbracket f(x) \rrbracket \leq \rrbracket f\left(x^{-1}\right) \rrbracket$ $(\forall x \in G)$, and for $x^{-1}$ instead of $x$, we get also the reverse inequality, therefore

$$
\llbracket f\left(x^{-1}\right) \rrbracket=\rrbracket f(x) \rrbracket \quad(\forall x \in G) .
$$

Furthermore $\llbracket f\left(x^{2}\right) \rrbracket={ }_{(4)}=\llbracket 2 f(x)+2 f\left(x^{-1}\right) \rrbracket \leq \llbracket 2 f(x) \rrbracket+\llbracket 2 f\left(x^{-1}\right) \rrbracket={ }_{(6)}=$ $4 \rrbracket f(x) \rrbracket \leq{ }_{(5)} \leq \llbracket f\left(x^{2}\right) \rrbracket$, so

$$
\llbracket f\left(x^{2}\right) \rrbracket=4 \rrbracket f(x) \rrbracket \quad(\forall x \in G) .
$$

Using ( JvN) for $\rrbracket \cdot \rrbracket^{2}$, we obtain $\llbracket f(x)-f\left(x^{-1}\right) \rrbracket^{2}=2 \rrbracket f(x) \rrbracket^{2}+2 \rrbracket f\left(x^{-1}\right) \rrbracket^{2}-\rrbracket f(x)+$ $f\left(x^{-1}\right) \rrbracket^{2}=(6),(4)=4 \rrbracket f(x) \rrbracket^{2}-\frac{1}{4} \rrbracket f\left(x^{2}\right) \rrbracket^{2}=(7)=4 \rrbracket f(x) \rrbracket^{2}-4 \rrbracket f(x) \rrbracket^{2}=0$, so 


$$
f\left(x^{-1}\right)=f(x) \quad(\forall x \in G),
$$

and together with (4)

$$
f\left(x^{2}\right)=4 f(x) \quad(\forall x \in G) .
$$

b) By (8) and Remark $2.2 \mathrm{~b})$, conditions $\left(\mathrm{C}^{\prime \prime}\right)$ and $\left(\mathrm{C}^{\prime \prime \prime}\right)$ are equivalent. An inspection of the part of the proof of ([4], Satz 1) following its formula (14) shows that in fact only condition $\left(\mathrm{C}^{\prime \prime \prime}\right)$ is needed for the simplification of its formula $(18)$, and $\left(\mathrm{C}^{\prime \prime \prime}\right)$ is strictly weaker than $(\mathrm{C})$ in our context as we shall see next.

Remark 3.3. a) Every quadratic mapping $f: G \longrightarrow E$ satisfies (3) since the additive group $(E,+)$ of our inner product space does not contain elements of order 2 ([11], p. 247, Lemma $4.1 \mathrm{a}), \mathrm{d})$, therefore also $(8),\left(\mathrm{C}^{\prime}\right),\left(\mathrm{C}^{\prime \prime}\right)$ and $\left(\mathrm{C}^{\prime \prime \prime}\right)$ $([7]$, p. $190,(2),(3),(5))$. Not every quadratic mapping satisfies $(\mathrm{C})$ of Theorem 1.1 b) (cf. [8], p. 36/37; worked out in [10]). Therefore, for quadratic mappings $f: G \longrightarrow E$, conditions $\left(\mathrm{C}^{\prime}\right),\left(\mathrm{C}^{\prime \prime}\right),\left(\mathrm{C}^{\prime \prime \prime}\right)$, and, e.g., $\left(\mathrm{C}^{\prime}\right) \wedge\left(\mathrm{C}^{\prime \prime}\right)$ are strictly weaker than $(\mathrm{C})$; remember Remark 2.2 a) for $(\mathrm{C}) \Longrightarrow\left(\mathrm{C}^{\prime}\right) \wedge\left(\mathrm{C}^{\prime \prime}\right) \wedge\left(\mathrm{C}^{\prime \prime \prime}\right)$ in general.

b) To use a commutativity substitute logically "below" the target assertion $(\mathrm{JvN})$ in Theorem $3.2 \mathrm{~b})$ is of course more adequate than $(\mathrm{C})$, which is "beyond" $\left(\mathrm{JVN}_{\mathrm{VN}}\right.$.

Remark 3.4. a) Other functional equations for the solutions of which $\left(\mathrm{C}^{\prime}\right)$ is strictly weaker that (C) are, e.g., the d'Alembert equation

$$
f: G \longrightarrow \mathbb{C}, f(e)=1, f(x y)+f\left(x y^{-1}\right)=2 f(x) f(y) \quad(\forall x, y \in G)
$$

([3], p. 339, (3); J. Lawrence, private communication), or

$$
f, g, h: G \longrightarrow \mathbb{C}, f(x y)+f\left(x y^{-1}\right)-2 f(x)=g(x) h(y) \quad(\forall x, y \in G)
$$

([1], p. 20/21, Remark 5).

b) On the other hand, there do exist functional equations for the solutions of which $(\mathrm{C})$ and $\left(\mathrm{C}^{\prime}\right)$ become equivalent, e.g.,

$$
\begin{gathered}
f: G \longrightarrow \mathbb{C}, e_{0} \in G, \quad e_{0} \neq e, \quad f\left(e_{0}\right)=1, \quad f(x y)+f(x y)^{-1}=2 f(x) f\left(e_{0} y\right) \\
(\forall x, y \in G)
\end{gathered}
$$

([2]), p. 51, Lemma 2).

At the end of this section, we present another stronger variant of Theorem 1.1 which is weaker than Theorem $3.2 \mathrm{~b}$ ) but the proof of which reveals a connection to one of the thirteen inequalities besides (I) associated with (JvN), namely to (14) below.

Theorem 3.5. If $(G, \cdot, e)$ is an arbitrary group, $(E,\langle\cdot, \cdot\rangle)$ an inner product space over $\mathbb{K}$, and $f: G \rightarrow E$ a solution of $(\mathrm{I})$ satisfying $\left(\mathrm{C}^{\prime}\right) \wedge\left(\mathrm{C}^{\prime \prime}\right)$, then $f$ is quadratic. 
Vol. 66 (2003) Inequalities associated with the Jordan-von Neumann functional equation 195

Proof. By Theorem 3.2 a), $f$ has properties (3), (8), and (9). (I) yields

$$
\begin{gathered}
\llbracket 2 f(x)+2 f(y) \rrbracket^{2}+\llbracket f\left(x y^{-1}\right) \rrbracket^{2}-2 \operatorname{Re}\left\langle 2 f(x)+2 f(y), f\left(x y^{-1}\right)\right\rangle \leq \llbracket f(x y) \rrbracket^{2} \\
(\forall x, y \in G) .
\end{gathered}
$$

Replacing $y$ by $y^{-1}$ and using (8) gives

$$
\llbracket 2 f(x)+2 f(y) \rrbracket^{2}+\llbracket f(x y) \rrbracket^{2}-2 \operatorname{Re}\langle 2 f(x)+2 f(y), f(x y)\rangle \leq \llbracket f\left(x y^{-1}\right) \rrbracket^{2} \quad(\forall x, y \in G) .
$$

By adding (10) and (11) and dividing by 2 we obtain

$$
\llbracket 2 f(x)+2 f(y) \rrbracket^{2} \leq \operatorname{Re}\left\langle 2 f(x)+2 f(y), f(x y)+f\left(x y^{-1}\right)\right\rangle \quad(\forall x, y \in G) .
$$

(12) together with $\operatorname{Re} \beta \leq|\operatorname{Re} \beta| \leq|\beta|(\forall \beta \in \mathbb{K})$ and the Cauchy-Schwarz Inequality (CSI) lead to

$$
\llbracket 2 f(x)+2 f(y) \rrbracket^{2} \leq \llbracket 2 f(x)+2 f(y) \rrbracket \cdot \llbracket f(x y)+f\left(x y^{-1}\right) \rrbracket \quad(\forall x, y \in G) .
$$

If $\llbracket 2 f(x)+2 f(y) \rrbracket>0$, we get from $(13) \rrbracket 2 f(x)+2 f(y) \rrbracket \leq \llbracket f(x y)+f\left(x y^{-1}\right) \rrbracket$, which holds also for $\llbracket 2 f(x)+2 f(y) \rrbracket=0$, so

$$
\llbracket 2 f(x)+2 f(y) \rrbracket \leq \llbracket f(x y)+f\left(x y^{-1}\right) \rrbracket \quad(\forall x, y \in G) .
$$

Let $x, y \in G$ be arbitrary and put $u:=x y, v:=x y^{-1}$. Then $\llbracket 2 f(x y)+$ $2 f\left(x y^{-1}\right) \rrbracket=\rrbracket 2 f(u)+2 f(v) \rrbracket \leq{ }_{(14)} \leq \rrbracket f(u v)+f\left(u v^{-1}\right) \rrbracket=\rrbracket f\left(x y x y^{-1}\right)+f\left(x y^{2} x^{-1} \rrbracket\right.$ $=\left(\mathrm{C}^{\prime \prime}\right),\left(\mathrm{C}^{\prime}\right)=\rrbracket f\left(x^{2}\right)+f\left(y^{2}\right) \rrbracket={ }_{(9)}=\llbracket 4 f(x)+4 f(y) \rrbracket$, briefly $\llbracket f(x y)+f\left(x y^{-1}\right) \rrbracket \leq$ $\llbracket 2 f(x)+2 f(y) \rrbracket$ and together with (14)

$$
\llbracket 2 f(x)+2 f(y) \rrbracket=\llbracket f(x y)+f\left(x y^{-1}\right) \rrbracket \quad(\forall x, y \in G) .
$$

Now (12), (15) and Lemma 3.1 with $a:=2 f(x)+2 f(y), b:=f(x y)+f\left(x y^{-1}\right)$ imply $2 f(x)+2 f(y)=f(x y)+f\left(x y^{-1}\right)$. Since $x, y \in G$ were arbitrary, this is $(\mathrm{JvN})$.

\section{Homogeneity of degree 2}

Remark 4.1. Theorem 3.2 a) guarantees (3), (8), and (9) for every solution $f: G \rightarrow E$ of (I). These three properties alone do not imply homogeneity of $f$ of degree 2 , i.e. the validity of

$$
f\left(x^{k}\right)=k^{2} f(x) \quad(\forall k \in \mathbb{Z}, \forall x \in G):
$$

Choose $(G, \cdot):=(\mathbb{R},+), E:=\mathbb{R}, f: \mathbb{R} \longrightarrow \mathbb{R}$ defined by $f(x):=4^{n_{x}}$ for $2^{n_{x}} \leq x<$ $2^{n_{x}+1}, f(0):=0, f(x):=f(-x)$ for $x<0$, where $n_{x} \in \mathbb{Z}$ is uniquely determined for every $x \in \mathbb{R}_{+}^{*}$. Then $f$ has the three properties above, (9) of course in the form $f(2 x)=4 f(x)(\forall x \in \mathbb{R})$, but $f(3 \cdot 1)=f(3)=4$ while $9 f(1)=9 \cdot 1=9$. On the other hand, with the aid of (I), (16) can be established, so that the solutions of (I) and those of $(\mathrm{JvN})$ share property (16): 
Theorem 4.2. If $(G, \cdot, e)$ is an arbitrary group, $(E,\langle\cdot, \cdot\rangle)$ an inner product space over $\mathbb{K}$, and $f: G \rightarrow E$ a solution of $(\mathrm{I})$, then we have for every $k \in \mathbb{Z}$

$$
f\left(x^{k}\right)=k^{2} f(x) \quad(\forall x \in G) .
$$

First proof. Let be $x \in G$ arbitrary and $C_{x}$ the subgroup of $G$ generated by $x$. Since $C_{x}$ is abelian, the restriction $f \mid C_{x}$ satisfies (I) on $C_{x}$ and condition $\left(\mathrm{C}^{\prime \prime}\right)$ and therefore is quadratic by Theorem $3.2 \mathrm{~b})$. Since $\left(f \mid C_{x}\right)(e)=f(e)=0$ by Theorem $3.2 \mathrm{a}), f \mid C_{x}$ is homogeneous of degree 2 ([11], p. 247, Lemma 4.1c). As $x \in G$ was arbitrary, (16) holds.

Second proof. (Induction on $k$; direct work with (I)). The statements $\left(16_{0}\right),\left(16_{-1}\right)$, $\left(16_{2}\right)$ are ensured by Theorem $\left.3.2 \mathrm{a}\right),(3),(8),(9)$, respectively, and $\left(16_{1}\right)$ is trivial. By (8), it is sufficient to consider the case $k \in \mathbb{N}^{0}$. So let be $k \in \mathbb{N}, k \geq 2$, and assume $\left(16_{\ell}\right)$ to hold for $\ell=0,1, \ldots, k-1$. For completing the proof, we have to show $\left(16_{k}\right)$ (second principle of induction). Case 1: $k$ is even, say $k=2 q$ with $q \geq 1$. Then $\left(16_{k}\right)$ easily follows from $\left(16_{q}\right)$ and (9). - Case $2: k$ is odd, say $k=2 q+1$ with $q \geq 1$. Then $k+1=2(q+1)$, and $\left(16_{k+1}\right)$ is obtained as in Case 1 . Let $x \in G$ be arbitrary. We first replace the pair $(x, y)$ in (I) by $\left(x^{k-1}, x\right)$, and $\left(16_{k-1}\right),\left(16_{k-2}\right)$ yield

$$
\llbracket k^{2} f(x) \rrbracket \leq \llbracket f\left(x^{k}\right) \rrbracket \quad(\forall x \in G) .
$$

Now $(x, y)$ is replaced in (I) by $\left(x^{k}, x\right)$, and $\left(16_{k-1}\right),\left(16_{k+1}\right)$ and squaring lead to

$$
\llbracket f\left(x^{k}\right) \rrbracket^{2} \leq\left((k-1)^{2}-2\right) \operatorname{Re}\left\langle f\left(x^{k}\right), f(x)\right\rangle+k^{2}(2 k+1) \rrbracket f(x) \rrbracket^{2} \quad(\forall x \in G) .
$$

Next, $(x, y)$ is substituted in (I) by $\left(x^{k}, x^{-1}\right)$, and $(8),\left(16_{k+1}\right),\left(16_{k-1}\right)$ similarly imply

$$
\llbracket f\left(x^{k}\right) \rrbracket^{2} \leq\left((k+1)^{2}-2\right) \operatorname{Re}\left\langle f\left(x^{k}\right), f(x)\right\rangle+k^{2}(1-2 k) \rrbracket f(x) \rrbracket^{2} \quad(\forall x \in G) .
$$

The combination $(2 k-1) \cdot(18)+(2 k+1) \cdot(19)$ provides

$$
\llbracket f\left(x^{k}\right) \rrbracket^{2} \leq k^{2} \operatorname{Re}\left\langle f\left(x^{k}\right), f(x)\right\rangle=\operatorname{Re}\left\langle f\left(x^{k}\right), k^{2} f(x)\right\rangle \quad(\forall x \in G) .
$$

From (20) and the CSI we get

$$
\llbracket f\left(x^{k}\right) \rrbracket^{2} \leq \llbracket f\left(x^{k}\right) \rrbracket \cdot \rrbracket k^{2} f(x) \rrbracket \quad(\forall x \in G) .
$$

If $f(x)=0$, we have equality in (21). If $f(x) \neq 0,(17)$ enforces $f\left(x^{k}\right) \neq 0$, so that (21) implies $\llbracket f\left(x^{k}\right) \rrbracket \leq \llbracket k^{2} f(x) \rrbracket$, and this is now true for all $x \in G$. Together with (17) we obtain

$$
\llbracket f\left(x^{k}\right) \rrbracket=\rrbracket k^{2} f(x) \rrbracket \quad(\forall x \in G) .
$$

Finally, (20), (22) and Lemma 3.1 with $a:=f\left(x^{k}\right), b:=k^{2} f(x)$ ensure $f\left(x^{k}\right)=$ $k^{2} f(x)(\forall x \in G)$, i.e., again $\left(16_{k}\right)$, and the second proof is complete.

Corollary 4.3. If $f: G \rightarrow E$ is a solution of (I) and $H$ a proper subgroup of $G$ such that $f$ is bounded on $G \backslash H$, then $f=\underline{0}$, so $f$ is quadratic. 
Proof. Let $\llbracket f(y) \rrbracket<c \quad(\forall y \in G \backslash H)$ for some $c \in \mathbb{R}_{+}^{*}$. Let $y \in G \backslash H$ be arbitrary and assume $f(y) \neq 0$. Then there would exist $n_{0} \in \mathbb{N}$ with $n_{0}^{2} \rrbracket f(y) \rrbracket \geq c$, i.e., by Theorem $4.2, \rrbracket f\left(y^{n_{0}}\right) \rrbracket \geq c$, so $y^{n_{0}} \in H$. $y^{n_{0}+1} \in H$ would imply $y=$ $y^{n_{0}+1}\left(y^{n_{0}}\right)^{-1} \in H$, contradicting the definition of $y$. Therefore $y^{n_{0}+1} \in G \backslash H$, hence $\left(n_{0}+1\right)^{2} \rrbracket f(y) \rrbracket=\rrbracket f\left(y^{n_{0}+1} \rrbracket<c \leq n_{0}^{2} \rrbracket f(y) \rrbracket\right.$, which is impossible. So $f(y)=0$. Since $y \in G \backslash H$ was arbitrary, we have

$$
f(y)=0 \quad(\forall y \in G \backslash H) .
$$

Now let $x \in H$ be arbitrary and $y \in G \backslash H$ (notice that $H \neq G$ ). $x y \in H$ would imply $y=x^{-1} x y \in H . x y^{-1} \in H$ would imply $y^{-1}=x^{-1} x y^{-1} \in H$, so $y \in H$. Therefore $x y, x y^{-1} \in G \backslash H$, and it follows from (I) and (23) that $\llbracket 2 f(x)+0-0 \rrbracket \leq \rrbracket 0 \rrbracket$, i.e. $f(x)=0(\forall x \in H)$, and together with (23) finally $f=\underline{0}$.

\section{Other situations where (I) does imply (JvN)}

Lemma 5.1. Let $G$ be an arbitrary group, $(E,\langle\cdot, \cdot\rangle)$ an inner product space over $\mathbb{K}$, and $f: G \rightarrow E$ a solution of $(\mathrm{I})$. Then we have:

a) If $x \in G$ has finite order, then $f(x)=0$.

b) If $G$ is a torsion group, specifically if $G$ is finite, then $f=\underline{0}$, so $f$ is quadratic.

Proof. a) There exists $m \in \mathbb{N}$ with $x^{m}=e$, so by (3) and Theorem $4.2 m^{2} f(x)=$ $f\left(x^{m}\right)=f(e)=0$, and the torsionfreeness of $(E,+)$ implies $f(x)=0$.

b) immediately follows from a).

Remark 5.2. Lemma 5.1 is now extended to a special class of possibly mixed groups $G$ (i.e., $G$ has elements of possibly infinite order). The concept is that of a special semidirect product of two groups $A$ and $B$. Since we wish that any two automorphisms of $B$ be summable in End $(B)$, we require that $B$ be an abelian group ([13], p. 52/53) and then accordingly use the additive notation $(B,+, 0)$ for $B$. If in addition $\left(A, \cdot, e_{A}\right)$ is a group and $g$ a given element of $\operatorname{Hom}((A, \cdot),(\operatorname{Aut}(\mathrm{B}), \circ))$, then the semidirect product of $A$ and $B$ with respect to $g$ is the group $G=A \times{ }_{g} B$ with underlying set $A \times B$ and the operation

$$
(a, b) \cdot\left(a^{\prime}, b^{\prime}\right):=\left(a a^{\prime}, b+g_{a}\left(b^{\prime}\right)\right) \quad\left(\forall(a, b),\left(a^{\prime}, b^{\prime}\right) \in A \times B\right),
$$

where $g_{a} \in \operatorname{Aut}(\mathrm{B})$ denotes the image of $a \in A$ under $g$. (In the literature, depending on the context, the notation $B \times{ }_{g} A$ is also used instead of $A \times{ }_{g} B$ ). $e:=\left(e_{A}, 0\right)$ turns out to be the identity element of $G$. If $g$ is constant, i.e., $g_{a}=i_{B}$ $\left(\forall a \in A\right.$ ), then $A \times{ }_{g} B$ coincides with the ordinary direct product of $A$ and $B$. If, however, $g$ is not constant, i.e., if there exists $a_{0} \in A$ with $g_{a_{0}} \neq i_{B}$, then there exists $b_{0} \in B$ with $g_{a_{0}}\left(b_{0}\right) \neq b_{0}$, and it follows that necessarily card $A \geq 2$, card $B \geq 3$, and that $A \times_{g} B$ is certainly non-abelian, even if $A$ is abelian. 
Theorem 5.3. Hypotheses: (i) $\left(A, ; e_{A}\right)$ is a torsion group, $\operatorname{card} A \geq 2$. (ii) $(B,+, 0)$ is an abelian group. (iii) $g \in \operatorname{Hom}(A, \operatorname{Aut}(\mathrm{B}))$ is such that card $g(A) \geq 2$ and $a \in A$, ord $a=n \geq 2 \Longrightarrow i_{B}+g_{a}+\ldots+g_{a^{n-1}}=\underline{0}$. (iv) $G=A \times_{g} B$. (v) $E$ is an inner product space over $\mathbb{K}$. Assertion: Every solution $f: G \rightarrow E$ of (I) is $\underline{0}$, so is quadratic.

Proof. 1) Let be $a \in A \backslash\left\{e_{A}\right\}, b \in B$ arbitrary, say ord $a=n \geq 2$. Then $(a, b)^{1}=$ $\left(a^{1}, i_{B}(b)\right)$, and if for $a k \in \mathbb{N}$ we have $(a, b)^{k}=\left(a^{k}, i_{B}(b)+g_{a}(b)+\ldots+g_{a^{k-1}}(b)\right)$, then by $(24)(a, b)^{k+1}=(a, b)^{k} \cdot(a, b)=\left(a^{k+1}, i_{B}(b)+\ldots+g_{a^{k-1}}(b)+g_{a^{k}}(b)\right)$, so

$$
(a, b)^{k}=\left(a^{k}, i_{B}(b)+g_{a}(b)+\ldots+g_{a^{k-1}}(b)\right) \quad(\forall k \in \mathbb{N}, k \geq 2) .
$$

By hypothesis (ii) and Remark 5.2, the automorphisms $i_{B}, g_{a}, \ldots, g_{a^{k-1}}$ of $B$ are summable in $\operatorname{End}(B)$, so by $(25)$

$$
(a, b)^{k}=\left(a^{k},\left(i_{B}+g_{a}+\ldots+g_{a^{k-1}}\right)(b)\right) \quad\left(\forall a \in A \backslash\left\{e_{A}\right\}, b \in B, k \geq 2\right) .
$$

ord $a=n \geq 2$, hypothesis (iii) and (26) imply $(a, b)^{n}=\left(a^{n}, \underline{0}(b)\right)=\left(e_{A}, 0\right)=e$, so that $(a, b)$ has order $n$ in $G$. If $f: G \rightarrow E$ is a solution of (I), Lemma 5.1a guarantees that $f(a, b)=0$. Let $\left.H:=\left\{e_{A}, b\right) ; b \in B\right\}$. So we have proved

$$
f(G \backslash H)=\{0\} .
$$

2) $H$ forms a subgroup of $G$ isomorphic to $B$ under the embedding $b \mapsto\left(e_{A}, b\right)$ $(b \in B)$. (By the way, this shows that $G$ may very well contain elements of infinite order, and since also $A$ is embeddable into $G$, this will then be a mixed group.) card $A \geq 2$ in hypothesis (i) ensures that $H$ is proper. By (27) and Corollary 4.3, $f=\underline{0}$, which ends the proof.

Of course, examples for the situation of Theorem 5.3 are of interest.

Example 5.4. The general dihedral group. Let $B$ be an abelian group of exponent $>2$ (i.e., there exists $b_{0} \in B$ with ord $b_{0}>2$ ), and $A:=\mathbb{Z}_{2}=\{1,-1\}$, the cyclic group in multiplicative notation. If $\chi_{B}$ is the mapping $b \mapsto b^{-1}(b \in B)$, then $\chi_{B} \neq i_{B}$, ord $\chi_{B}=2$ in $\operatorname{Aut}(\mathrm{B}) . g \in \operatorname{Hom}(A, \operatorname{Aut}(\mathrm{B}))$ is defined by $g_{1}:=i_{B}$, $g_{-1}:=\chi_{B}$. Now the dihedral group associated to $B$ is $\operatorname{Dih} B:=\mathbb{Z}_{2} \times_{g} B$. In our situation, Dih $B$ is always non-abelian. For $B=Z_{3}$ we get $\operatorname{Dih} B=S_{3}$, the smallest non-abelian group. For $B=\mathbb{Z}_{n}(n \geq 3)$ we obtain as $\operatorname{Dih} B$ the finite dihedral groups, for $B=\mathbb{Z}$ the infinite dihedral group, and for $B=\mathbb{R}$ the group of rigid motions of $\mathbb{R}^{1}$. Hypotheses (i), (ii), (iv) of Theorem 5.3 clearly are satisfied, but also (iii): $a=-1 \in \mathbb{Z}_{2}$, ord $a=2, i_{B}+g_{a}=i_{B}+g_{-1}=i_{B}+\chi_{B}=\underline{0}$ since $\left(i_{B}+\chi_{B}\right)(x)=x+(-x)=0(\forall x \in B)$. So the assertion of Theorem 5.3 holds in all these cases.

Example 5.5. Let $(B,+, \cdot)$ be a ring with $1 \neq 0$, not necessarily commutative, having no zero divisors, and let $A$ be a torsion subgroup of the group $U(B)$ of units of $B, \operatorname{card} A \geq 2 . g \in \operatorname{Hom}(A, \operatorname{Aut}(\mathrm{B},+))$ is defined by $g_{a}(b):=a b(\forall b \in B$, 
Vol. 66 (2003) Inequalities associated with the Jordan-von Neumann functional equation 199

$a \in A$ ) and $G:=A \times{ }_{g} B$. Now the hypotheses (i), (ii), (iv) of Theorem 5.3 are fulfilled, but also (iii): If $a_{0} \in A \backslash\{1\}$, then $g_{a_{0}}(1)=a_{0} \neq 1=g_{1}(1)$, so $g_{a_{0}} \neq g_{1}$, so card $g(A) \geq 2$. Let $a \in A$, ord $a=n \geq 2$. Then $0=a^{n}-1=(a-1)\left(1+\ldots+a^{n-1}\right)$, and $a-1 \neq 0$ implies $1+a+\ldots+a^{n-1}=0$, so $\left(1+a+\ldots+a^{n-1}\right) b=0$, i.e., $\left(i_{B}+g_{a}+\ldots+g_{a^{n-1}}\right)(b)=0(\forall b \in B)$, i.e., $i_{B}+g_{a}+\ldots+g_{a^{n-1}}=\underline{0}$. So Theorem 5.3 is applicable.

a) If our ring $B$ has characteristic $\neq 2$, then $A=\{1,-1\}$ becomes possible, and we return to Example 5.4; notice that $b \mapsto(-1) b(b \in B)$ is nothing else than $\chi_{B}$.

b) If $B=\mathbb{C}$, then $A$ may be the locally cyclic group $\mathbb{Q} / \mathbb{Z}$ of all roots of unity of all orders or a subgroup thereof.

Example 5.6 (modification of Example 5.5). For the ring $(B,+, \cdot)$ we now allow the existence of zero divisors, leave everything else unchanged, choose $A, g$, and $G$ as before but require now the condition

$$
a \in A, a \neq 1 \Longrightarrow(a-1) \in U(B) .
$$

The only crucial point is again hypothesis (iii) of Theorem 5.3: Let $a \in A$, ord $a=$ $n \geq 2$. We have again $0=a^{n}-1=(a-1)\left(1+\ldots+a^{n-1}\right)$. This and (28) imply $1+\ldots+a^{n-1}=(a-1)^{-1}\left(a^{n}-1\right)=0$ and then, as in Example 5.5 $i_{B}+\ldots+g_{a^{n-1}}=\underline{0}$, and Theorem 5.3 can be applied in this situation. An illustration: $n \in \mathbb{N}, B:=\mathbb{F}^{n \times n}$, the ring of all $n \times n$ matrices over the field $\mathbb{F}$, $A$ a torsion subgroup of $U(B)=G L(n, \mathbb{F})$ satisfying (28). Specifically, if $n=2$, $\mathbb{F}=\mathbb{R}, a_{r}:=\left(\begin{array}{rr}\cos (2 \pi r) & -\sin (2 \pi r) \\ \sin (2 \pi r) & \cos (2 \pi r)\end{array}\right)(\forall r \in \mathbb{Q}), A:=\left\{a_{r} ; r \in \mathbb{Q}\right\}$ is the image of $\varphi \in \operatorname{Hom}((\mathbb{Q},+),(A, \cdot))$ given by $\varphi(r):=a_{r}(\forall r \in \mathbb{Q}) . A$ is a torsion group and satisfies $(28)$ since $(\cos (2 \pi r)-1)^{2}+(\sin (2 \pi r))^{2}=2-2 \cos (2 \pi r) \neq 0$ for $r \in \mathbb{Q} \backslash \mathbb{Z}$. Of course, $A$ is isomorphic to $\mathbb{Q} / \mathbb{Z}$.

Acknowledgement. The author wishes to thank the referees for their valuable suggestions which lead to essential improvements of the text.

\section{References}

[1] J. AczÉL, J. K. Chung And C. T. NG, Symmetric second differences in product form on groups, in: Th. Rassias (ed.), Topics in Math. Analysis, 1-22, World Sci. Publ. Co., 1989.

[2] D. DACić, On the sine functional equation for groups, Mat. Vesnik 9 (24) (1972), 49-53.

[3] R. DAcić, The cosine functional equation for groups, Mat. Vesnik 6 (21) (1969), 339-342.

[4] A. Gilányi, Eine zur Parallelogrammgleichung äquivalente Ungleichung, Aequationes Math. 62 (2001), 303-309.

[5] A. GilánYI, On inequalities derived from the square-norm equation, Report of the 38th International Symposium on Functional Equations, June 11-18, 2000, Noszvaj, Hungary, Aequationes Math. 61 (2001), 289.

[6] A. GilÁNYI, Remark 12, Report of the 39th International Symposium on Functional Equations, August 12-18, 2001, Sandbjerg, Denmark, Aequationes Math. 64 (2002), 190-191. 
[7] M. Hosszú, A remark on the square norm, Aequationes Math. 2 (1969), 190-193.

[8] M. Hosszú, und M. Csikós, Normenquadrat über Gruppen, Zbornik Radova Mat. Inst. Beograd (N.S.) 1 (9) (1976), 35-39.

[9] Pl. Kannappan, The functional equation $f(x y)+f\left(x y^{-1}\right)=2 f(x) f(y)$ for groups, Proc. Amer. Math. Soc. 19 (1968), 69-74.

[10] J. RÄтz, Remark 4, Report of the 21th International Symposium on Functional Equations, Aequationes Math. 26 (1984), 261-262.

[11] J. Räтz, On orthogonally additive mappings, II, Publ. Math. Debrecen 35 (1988), 241-249.

[12] J. RÄтz, On inequalities associated with the Jordan-von Neumann functional equation, Report of the 39th International Symposium on Functional Equations, August 12-18, 2001, Sandbjerg, Denmark, Aequationes Math. 64 (2002), 180.

[13] M. SuZuki, Group Theory I, Grundlehren der mathematischen Wissenschaften, Band 247, Springer, Berlin-Heidelberg-New York, 1982.

\section{J.Rätz}

Mathematisches Institut

Universität Bern

Sidlerstrasse 5

CH-3012 Bern

Switzerland

Manuscript received: January 18, 2002 and, in final form, August 20, 2002.

To access this journal online:

(40) http://www.birkhauser.ch 